\title{
Homöopathische Arznei, homöopathische Anamnese, Empathie oder gar kein Effekt?
}

\author{
Rainer Lüdtke
}

Karl und Veronica Carstens-Stiftung, Essen, Deutschland

Es gibt in der Medizin Themen, bei denen sich jeder Arzt und Patient berufen fühlt mitzureden. Ähnlich wie es in Deutschland geschätzte 40 Millionen Fußball-Bundestrainer gibt, scheint es auch 40 Millionen Experten für Homöopathie und ebenso viele Fachleute für Placebo und Placeboeffekte zu geben. Doch genauso, wie die meisten von uns keine tiefen Einsichten in fußballtaktische Feinheiten haben, fehlt uns ein Verständnis für Details und Nuancen zu wissenschaftlichen Erkenntnissen in Bezug auf die letzteren beiden Themen. In der Regel kulminiert die sogenannte Expertenmeinung zur Homöopathie in der Phrase «Ist doch alles nur Placebo!» und zum Placeboeffekt in der Behauptung: «Man muss halt nur dran glauben!». Wer etwas tiefer in die Materie eingestiegen ist, formuliert: «Die Chemie muss stimmen zwischen Arzt und Patient!» oder «Der Arzt müsste einfach nur mehr Zeit für den Patienten haben». Nur wenige realisieren, dass hinter diesen Aussagen durchaus sehr verschiedene - vielleicht sogar konkurrierende - Konzepte stehen, wie Placeboeffekte hervorgerufen werden können, und dass diese Konzepte durchaus unterschiedliche Interpretationen zur Folge haben, wie die Homöopathie wirkt. Das ist eine Diskussion, die schon seit über 200 Jahren geführt wird, in letzter Zeit aber durchaus neu belebt wurde, auch durch erstaunliche - manchmal sogar scheinbar paradoxen - Ergebnisse.

Für die meisten von uns schwingt beim Begriff «Placebo» auch immer die Bedeutung von Täuschung, Bluff und Augenwischerei mit - was voraussetzt, dass es dem Patienten nicht bewusst ist, ein Placebo zu bekommen. Ein Placebo zu geben und den Patienten gleichzeitig davon in Kenntnis zu setzen, dass es ein Placebo ist, scheint sinnlos. Glaubten wir! - bis vor kurzem Kaptchuk et al. [1] eine Aufsehen erregende Studie veröffentlichte, nach der eine offene Placebogabe im Vergleich zum therapeutischen Abwarten bei Patienten mit Reizdarmsyndrom wirksam ist. Obwohl die Patienten ausführlich darüber aufgeklärt wurden, was ein Placebo ist und was den
Placeboeffekt vermutlich ausmacht, reagierten sie auf die Placebogabe mit erheblichen Verbesserungen ihrer Symptome. Zwei Interpretationen stehen im Raum: Entweder ist es das Ritual der Arzneigabe selbst, das den gesundheitsfördernden Effekt völlig unabhängig von der inhaltlichen Konnotation auslöst. Oder Robert Ader, einer der Nestoren der Placeboforschung, hat Recht, indem er behauptet, die Studie zeige, «dass die Kraft der Suggestion durch Sachverständige das Wissen, dass ein Placebo pharmakologisch inaktiv ist, überschreiben kann» [2].

Beide Interpretationen haben Auswirkungen darauf, wie Homöopathie wirken kann. Gemäß der ersten Interpretation wird dem Ritual der Arzneigabe eine entscheidende Rolle bei der Auslösung eines gesundheitsfördernden Effekts zugebilligt. Gerade dieser Prozess ist aber in der Homöopathie besonders ausgeprägt und eigenartig. Er wird von vielen Homöopathen geradezu zelebriert, entweder indem die ersten Globuli dem Patienten vom Arzt persönlich unter die Zunge gelegt werden oder indem der Patient über mehrere Tage vertröstet wird, bis der Arzt glaubt, das Simillimum gefunden zu haben. Ist es also gerade das absonderliche, geradezu anachronistische ritualisierte Vorgehen, das den Homöopathen erfolgreich macht?

Oder ist es die ärztliche Empathie, die wirkt? Eine Vermutung, die durch die zweite Interpretation der KaptchukErgebnisse gestützt würde, wobei es sich um eine durchaus paternalisitische Interpretation von Empathie handelt - nämlich der Vorstellung, dass der Arzt den Patienten kraft seines Sachverstands und seiner Autorität beeinflussen kann. Das allerdings wäre schwer mit den bisherigen Vorstellungen zu vereinbaren, dass es gerade die kooperative, partnerschaftliche Beziehung in der Arzt-Patienten-Interaktion ist, die den Weg für eine Genesung ebnet [3, 4]. Gerade erst haben Forscher der Universitäten Freiburg i.Br., Aachen und Witten diese Sichtweise für die Komplementärmedizin noch einmal

\section{KARGER \\ Fax +497614520714 \\ Information@Karger.de}

www.karger.com (c) 2011 S. Karger GmbH, Freiburg

$1661-4119 / 11 / 0182-0064 \$ 38.00 / 0$

Accessible online at:

www.karger.com/fok
Dipl.-Stat. Rainer Lüdtke

Karl und Veronica Carstens-Stiftung

Am Deimelsberg 36, 45276 Essen, Deutschland

r.luedtke@carstens-stiftung.de 
besonders betont. In einer Studie, in der eigentlich ein Fragebogen zur Messung der Empathie validiert werden sollte, fanden sie ein bemerkenswertes Nebenergebnis: «Dieses lässt vermuten, dass emotional-ethische und Verhaltensaspekte der Empathie in der Arzt-Patienten-Interaktion unterschiedliche Rollen spielen, je nachdem unter welcher Erkrankung der Patient leidet und wie er behandelt wird. Für komplementär Behandelte (...) scheinen dabei die Verhaltensaspekte wesentlich wichtiger zu sein, nicht (...) emotionale und ethische Aspekte (...)»[5].

All dieses sollte man bedenken, wenn man die neuesten Ergebnisse aus der Southamptoner Arbeitsgruppe um Sarah Brien und George Lewith diskutiert. Diese hatten in einer randomisierten Therapiestudie zur rheumatoiden Arthritis [6] fünf Gruppen von Patienten gebildet. Drei davon erhielten eine vollständige homöopathische Anamnese und danach entweder eine homöopathische Einzelmittelbehandlung, ein homöopathisches Komplexmittel oder ein Placebo. Die bei- den verbleibenden Gruppen bekamen keine homöopathische Anamnese und wurden entweder mit dem Komplexmittel oder einem Placebo behandelt. Ein Therapieerfolg stellte sich vornehmlich bei den Patienten ein, die auch eine homöopathische Anamnese erhalten hatten - wobei der Effekt allerdings statistisch nicht signifikant war.

Vor dem Hintergrund des oben Gesagten ist vor pauschalierenden und vereinfachenden Interpretationen nur zu warnen. Diese Studie zeigt beileibe nicht, dass allein «Zuhören» und «Zeit haben» ausreicht, um gesundheitsfördernde Effekte hervorzurufen. Empathie, Kontrolle und Ritual sind Elemente, die andere Erklärungen liefern. Und diese könnten durchaus «spezifisch» genannt werden. Nach Linde [7] ist es dafür notwendig, dass ein klarer Wirkmechanismus benannt werden kann, der Effekte hervorruft, die von spezifischen Bedingungen abhängt. Die Wissenschaft arbeitet gerade daran, Teile der Placeboeffekte als spezifisch zu entlarven. Und damit 40 Millionen Experten eines Besseren zu belehren.

\section{Literatur}

1 Kaptchuk TJ, Friedlander E, Kelley JM, Sanchez MN, Kokkotou E, Singer JP, Kowalczykowski M, Miller FG, Kirsch I, Lembo AJ: Placebos without deception: a randomized controlled trial in irritable bowel syndrome. PLoS One 2010;5:e15591.

2 Ader R: Placebos without deception: a comment. PLoS One 2011; available from www.plosone.org/ article/comments/info $\% 3$ Adoi $\% 2$ F10.1371\%2 Fjournal.pone.0015591.

3 Bikker AP, Mercer SW, Reilly D: A pilot prospective study on the consultation and relational empathy, patient enablement, and health changes over 12 months in patients going to the Glasgow Homoeopathic Hospital. J Altern Complement Med 2005;11:591-600.
4 MacPherson H, Mercer SW, Scullion T, Thomas KJ: Empathy, enablement, and outcome: an exploratory study on acupuncture patients' perceptions. J Altern Complement Med 2003;9:869-876.

5 Wirtz M, Boecker M, Forkmann T, Neumann M: Evaluation of the 'Consultation and Relational Empathy' (CARE) measure by means of Raschanalysis at the example of cancer patients. Patient Educ Couns 2011; doi: 10.1016/j.pec.2010.12.009
6 Brien S, Lachance L, Prescott P, McDermott C, Lewith G: Homeopathy has clinical benefits in rheumatoid arthritis patients that are attributable to the consultation process but not the homeopathic remedy: a randomized controlled clinical trial. Rheumatology (Oxford) 2010; doi: 10.1093/ rheumatology/keq234.

7 Linde K: Der spezifische Placeboeffekt. Bundesgesundheitsbl Gesundheitsforsch Gesundheitsschutz 2006;49:729-735. 